Research Article

iMedPub Journals

www.imedpub.com

\section{Possibilities of Molecular-Genetic Tests in Periodontology}

\section{Abstract}

Treatment protocol for inflammatory periodontal disease therapy, as a rule, includes local and general antibiotic medication targeted at periopathogenic bacteria. This paper reviews chronic periodontitis medication efficacy of the new bacteriophage dental gel for local application in complex treatment of chronic periodontitis.

Keywords: Bacteriophage; Chronic periodontitis; Antibiotic therapy; Periopathogenic bacteria

Received: February 01, 2018; Accepted: February 05, 2018; Published: February 09, 2018

\section{Topicality of the Research}

Inflammatory periodontal diseases are multifactorial diseases. The main risk factors for their development include genetic predisposition, excessive immune response of the human body and environmental factors (microflora of the oral cavity). Modern researchers prefer genetic risk factors in the pathogenesis of periodontal diseases. Brayan S Michalowicz, based on the obtained clinical studies of families, showed that susceptibility to early forms of periodontal disease depends on the host's genotype. The inherited deficiencies of phagocytic cells contribute to the development of the disease. Later, in 2002, Brayan S Michalowicz and colleagues assessed the condition of periodontium in 110 twins; the average age of the subjects was 40 years. The authors concluded that chronic periodontitis is inherited in $50 \%$ cases. A decrease in bone tissue by $82 \%$ is associated with genetic factors. Studies by Marja L. Laine, Bruno $\mathrm{G}$ Loos confirmed the leading role of genetic polymorphisms in the development of chronic forms of periodontal disease.

None of the variants of the gene has yet manifested itself, as definitely predisposing to periodontitis. Studies are conducted in 3 directions: 1 - study of candidate genes; 2 - study of associations of the genome; 3 - analysis of the genetic variants of the host (SNP) [1].

Results of numerous domestic and foreign authors studies show, that the periodontal diseases leading etiological factor are periopathogenic bacteria [2-5]. Most of them are anaerobes, characterized by high adhesiveness, invasiveness and toxicity. Periopathogenic bacteria are united in so-termed "complexes", of which the most aggressive for periodontal tissues are "pink"

\section{Elena Oleinik ${ }^{1 *}$, Roman Ilyk ${ }^{4}$ \\ Julia Tkachenko², Rafik Kamalov ${ }^{3}$, Konstantin Janishevskii', Vasiliy Lysohor ${ }^{1}$, Olga Futomskaya ${ }^{1}$ and Lubomir Janishevskii ${ }^{1}$}

1 Department of Therapeutic Dentistry, Private Higher Educational Establishment, Kyiv Medical University, Ukraine

2 Departament of Pediatric Dentistry, Orthodontics and Implantology, Kharkiv Medical Academy of Postgraduate Education, Ukraine

3 Department of Dentistry, PL Shupiyk National Medical Academy of Postgraduate Education, Ukraine

4 Professor, Department of orthopedic medicine, National Medical University Lviv, named after Danylo Galyzkiy

\section{*Corresponding author: Elena Oleinik anelee1970@gmail.com}

Department of Therapeutic Dentistry, Private Higher Educational Establishment, Kyiv Medical University, Ukraine.

\section{Citation: Oleinik E, Ilyk R, Tkachenko J, Kamalov R, Janishevskii K, et al. (2018) Possibilities of Molecular-Genetic Tests in Periodontology. J Clin Epigenet. Vol.4 No.1:3}

(exponent - Actinobacillus actinomycetemcomitans) and "red" (representatives - Porphyromonas gingivalis, Tannerella forsythia, Treponema denticula) [4-7]. Thus, in periodontal practice, for their suppression, as a rule, broad-spectrum antibiotics are used. Ultimately, the pathological processes treatment with antibiotics can lead to a change in T-lymphocytes level in the body, but indirectly, after the disappearance of the inflammatory reaction.

Despite the proven clinical effectiveness, the antibiotics administration entails a number of problems, such as their imperfect efficacy due to the periopathogenic bacteria 
increased resistance and formation of resistant strains of the microorganisms, as well as pronounced side effects $[8,9]$. In view of this circumstance, we see an alternative to the antibacterial treatment of inflammatory periodontal diseases are bacteriophages, which therapeutic effect was discovered almost one hundred years ago. The possibility of phagotherapy administration in the periodontal diseases treatment was suggested by a number of authors [10-12].

The antibacterial effect of bacteriophages is due to the introduction of the phage genome into a bacterial cell with its subsequent multiplication and lysis of the infected cell. The bacteriophages, released into the external environment as a result of lysis, repeat the cycle of introduction into other bacterial cells, and lyse them, acting until the pathogens are eliminated completely in the inflammation site.

So, topicality of the research of bacteriophages therapy efficacy as an alternative to antibiotic therapy in the complex treatment of periodontal diseases is obvious.

Research objective: study efficacy of the treatment-andprophylactic preparation Fagodent aimed at inflammatory periodontal disease therapy.

\section{Materials and Methods}

Studies within the pilot project of the Belgorod region were conducted on the basis of the Interregional Centre of Dental Innovations (ICDI) of the National Research University "Belgorod State University" in the city of Belgorod. During the study, the ethical standards set out in the Helsinki Declaration (1964), modified by the World Assembly 41 (Hong Kong,) were respected, information consents were signed.

A total of 38 patients aged from 32 to 55 years, who attended for professional hygiene of the oral cavity, as well as for periodontal treatment, passed examination and were admitted for treatment at the ICDI NRU "BelSU" with diagnosis "chronic generalized moderately severe periodontitis". All patients received the standard periodontal treatment. Two groups were formed: group $1-16$ subjects, who received the complex periodontal treatment with the medication Fagodent; group $2-22$ subjects, who received for periodontal treatment antibiotic Clindamycinum of lincosamides and most effective against anaerobic bacteria, as Bacteroides. The medicine was administered for 7 days according to the scheme proposed by the German Dental Society of Periodontologists. The clinical examination included: anamnesis, patient complaints and oral examinations. Additional methods of investigation were determining the hygienic state of the oral cavity using a simplified oral hygiene index (SOHI) after GreenWermillion, gingivitis index of RMA, as well as X-ray imaging.

Periopathogenic complexes were defined with test Micro-IDent 11 plus (Hain Lifescience, Germany) before and 1 month after Fagodent treatment. This diagnostic method is based on the reverse hybridization principle, which involves DNA amplicon denaturation and applying the obtained sample to a nitrocellulose strip containing specific probes of 11 periodontogenic agents, a control probe for the isolated DNA and the conjugate control.
Amplicons associated with the complentary probe were visualized after addition of the streptavidin-alkaline phosphatase conjugate. The results were read after the provided template.

In virtue of complaints, the disease anamnesis, objective examination data, the periodontal tissues condition was evaluated, a diagnosis was established and a comprehensive treatment plan was developed using the domestic medication Fagodent. Registration of the hygienic and clinical indices was made before and 1 month after the treatment.

Treatment of chronic generalized periodontitis assumed compliance with general medical principles: complexity, individuality, consistency. Subjects were pre-appointed correction of individual oral hygiene and occupational hygiene session. After the acute manifestations of the inflammatory process remitted, the patients received curettage of periodontal pockets with injection of Fagodent from the syringe cannula. After 15 minutes, the procedure was finished, the mouth was not rinsed. The test medication was also prescribed for home applications during 2 weeks.

The treatment results were estimated by analysis of the main subjects' complaints and objective examination data.

\section{Results and Discussion}

Before the treatment, patients complained of painful and bleeding gums, discoloration, swelling, bad breath, discomfort due to calculus, exposure of the teeth necks, teeth mobility. One month after treatment with "Fagodent", reduction of pain disorder, bleeding, periodontal pockets pyorrhoea was noted. However, it should be acknowledged that treatment with the gel with bacteriophages has not give the expected result for elimination of such factors as "bad" breath, "swelling of the oral mucosa". Data of the objective examination of patients before and after the complex periodontal treatment are presented in Table 1.

The state of oral hygiene was assessed after $\mathrm{OHI}-\mathrm{S}$. According to the data received, individual oral hygiene before the treatment in all subjects with periodontal disease was unsatisfactory. OHI-S mean value before treatment was $4.4 \pm 0.37$ (group I), and after professional teeth cleaning $-2.46 \pm 0.40$, speaking for satisfactory oral hygiene. However, OHI-S mean value in group II was $4.1 \pm$ $0.26,1$ month after $-1.5 \pm 0.52$.

Admittedly, on the top of the administered therapy, we noted a tendency to inflammatory processes reduction in patients of both groups after PMA-index. The data of the subject index examination before and after the complex periodontal treatment are presented in Table 2.

Analyzing index of RMA in subjects of Group I and Group II received 1 month after the periodontal treatment, we can conclude that both broad-spectrum antibiotics and bacteriophage "Fagodent" stop the inflammatory process. The results of test "Micro-IDent plus" are of special interest. 15 subjects from group I (94\%) and 20 subjects from group II (91\%) showed superthreshold markers of "red" and "orange" complexes. Figure 1 shows results of 
the test "Micro-IDent" in subject P. before the treatment with agodent" and Figure 2 shows results of the test "Micro-IDent" in subject B. before treatment with broad-spectrum antibiotics.

After 1 month, repeated analysis of Micro-IDent 11 plus detected only "traces" of dangerous periopathogenic complexes markers both in subjects from group I and from the control group. Results of the study are shown in Figures 3 and 4.
However, $59 \%$ of subjects (13 persons) who was taking antibiotic Clindamycinum had side effects of the medication, specifically: disorders of the gastrointestinal tract associated with partial loss of useful microflora (62\% - 8 persons), allergic reactions ( $31 \%$ - 4 persons), arterial hypotension (7\% - 1 person). Similar complaints from patients using "Fagodent" were not reported [13-15].

Table 1 Dynamics of the inflammatory process evaluation criteria in patients with CGMSP.

\begin{tabular}{|c|c|c|c|c|c|c|}
\hline \multirow[t]{2}{*}{ Symptoms } & \multicolumn{2}{|c|}{ Group I n=16 } & \multirow[t]{2}{*}{$\mathbf{p}$} & \multicolumn{2}{|c|}{ Group II n=22 } & \multirow[t]{2}{*}{$\mathbf{P}$} \\
\hline & Before treatment & 1 month after & & Before treatment & 1 month after & \\
\hline Pain & $5.86 \pm 0.83$ & $2.7 \pm 0.71$ & $p<0.05$ & $5.2+0.64$ & $2.2+0.56$ & $p<0.05$ \\
\hline Haemorrhage & $7.57 \pm 0.65$ & $3.43 \pm 0.95$ & $p<0.05$ & $8.6+0.62$ & $5.6+0.55$ & $p<0.01$ \\
\hline Swelling & $6.57 \pm 1.04$ & $4.7 \pm 0.97$ & $p<0.01$ & $7.7+0.4$ & $5.9+0.26$ & $p<0.01$ \\
\hline Pyorrhoea & $4.6 \pm 1.3$ & $1.3 \pm 0.71$ & $p<0.05$ & $5.2+0.6$ & $3.1+0.46$ & $p<0.05$ \\
\hline Discoloration & $7.7 \pm 0.75$ & $3.43 \pm 0.97$ & $p<0.05$ & $6.9+0.55$ & $3.7+0.69$ & $p<0.05$ \\
\hline Bad breath & $6.7 \pm 1.1$ & $1.7 \pm 0.57$ & $p<0.05$ & $5.9+1.2$ & $2.3+0.36$ & $p<0.05$ \\
\hline Masticatory efficiency & $8.3 \pm 0.2$ & $3 \pm 0.87$ & $p<0.05$ & $8.8+0.6$ & $4.2+0.59$ & $p<0.05$ \\
\hline
\end{tabular}

Table 2 Dynamics of periodontal status according to the index evaluation in patients with CGMSP in the course of complex treatment with the gel "Fagodent".

\begin{tabular}{|c|c|c|c|c|}
\hline Index & Before treatment & $\mathbf{P}$ & 1 month after & $\mathbf{P}$ \\
\hline \multicolumn{5}{|l|}{ RMA } \\
\hline Group I (n=16) & $55 \pm 0.52$ & $p<0.05$ & $28 \pm 0.62$ & $p<0.05$ \\
\hline Group II $(n=22)$ & $51 \pm 0.24$ & $p<0.01$ & $32 \pm 0.46$ & $p<0.01$ \\
\hline
\end{tabular}

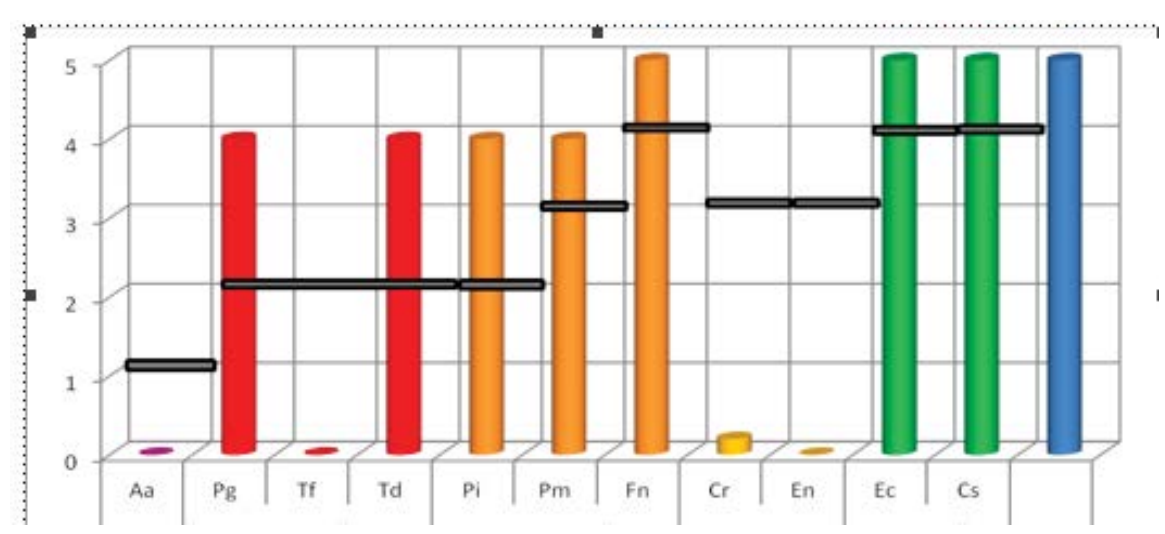

Figure 1 Dynamics of the inflammatory process evaluation criteria in patients with CGMSP.

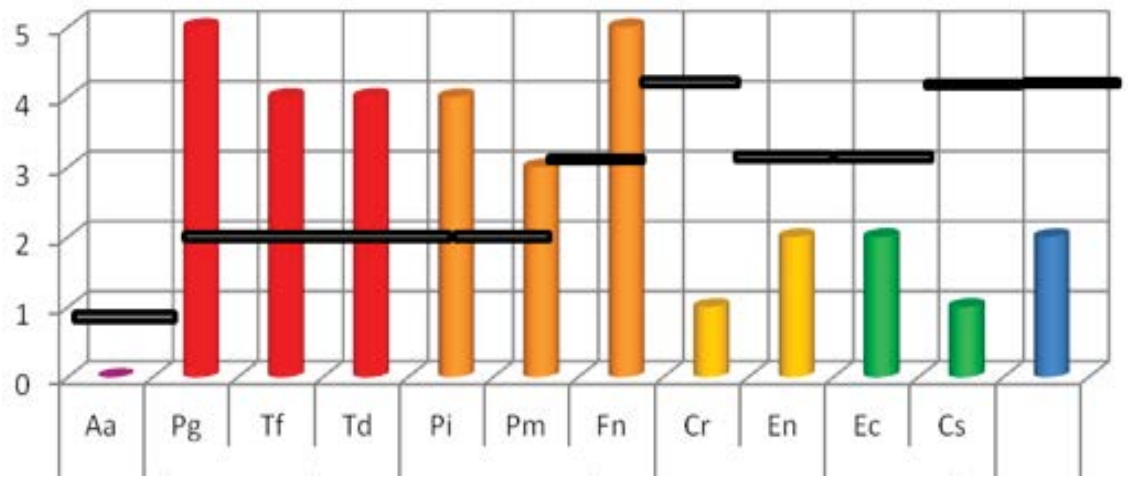

Figure 2 Results of the analysis Micro-IDent 11 plus in subject B. before treatment with antibiotic Clindamycinum. 


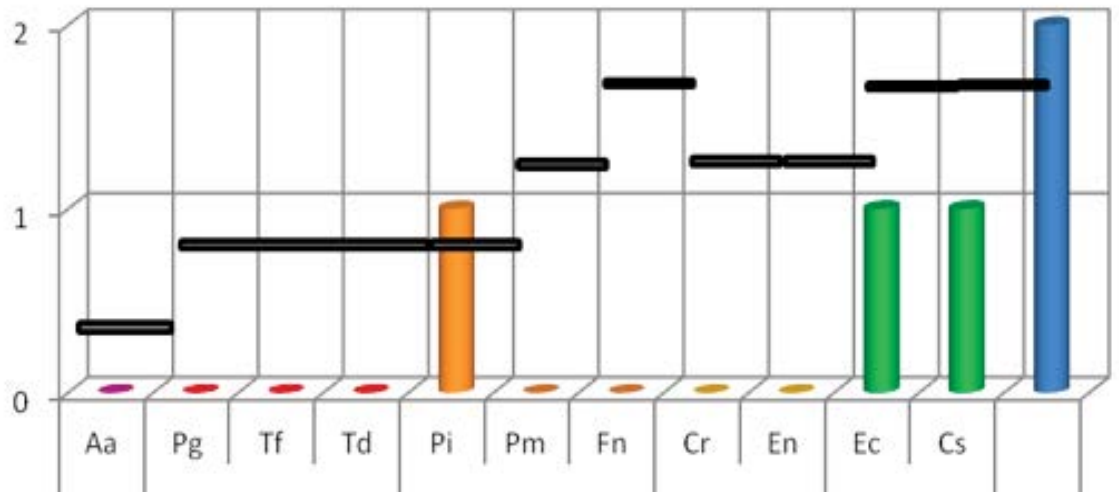

Figure 3 Results of the analysis Micro-IDent 11 plus in subject P. 1 month after treatment with "Fagodent".

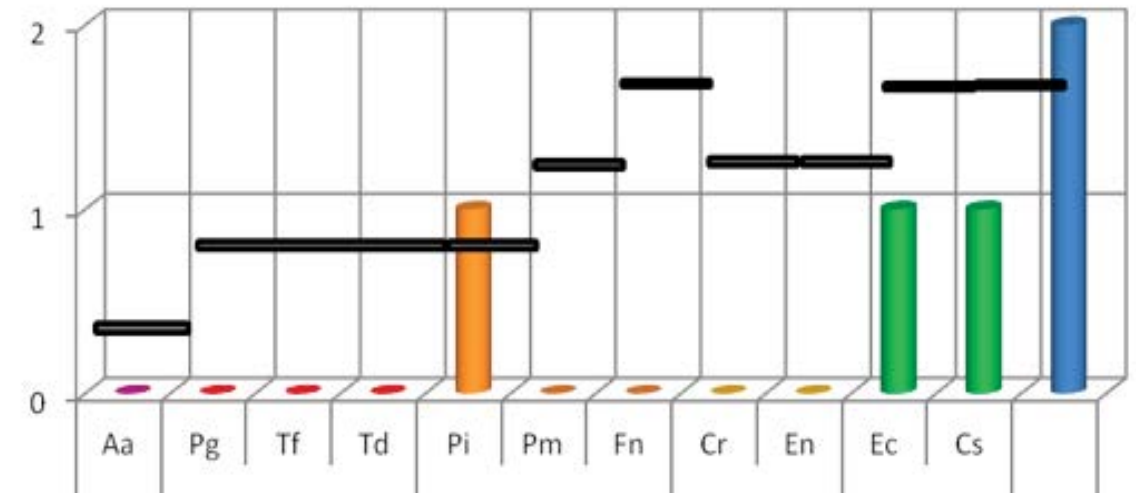

Figure 4 Results of the analysis Micro-IDent 11 plus in subject B. 1 month after treatment with antibiotic Clindamycinum.

\section{Conclusion}

It should be noted that, as a result of the groups of subjects with chronic generalized periodontitis of moderate severity studies, effectiveness in complex treatment of "Fagodent" based on virulent bacteriophages is shown. The proposed scheme of professional and individual administration of "Fagodent" can be used as an alternative to administration of the broad-spectrum

\section{References}

1 Nibali L, Di lorio A, Tu YK, Vieira AR (2017) Host genetics role in the pathogenesis of periodontal disease and caries. J Clin Periodontology 44: S52-S78.

2 Barer GM (2008) Therapeuthic stomatology. Moscow: GEOTARMedia.

3 https://www.wiley.com/en-us/Periodontal+Medicine+and+Systems +Biology-p-9781405122191

4 Brown MR, Gilbert P (1993) Sensitivity of biofilms to antimi- crobial agents. J Appl Bacteriol 74: 87S-97S.

5 Mitchell HL, Dashper SG, Catmull DV, Paolini RA, Cleal SM (2010) Treponema denticola biofilm-induced expression of a bacteriophage, toxin-antitoxin systems and transposases. Microbiology 156: 774-788. antibiotics. The clinical effect is the same, and the probability of complications is much lower.

\section{Conflict of Interest and Source of Fund- ing Statement}

The authors openly state that there is no conflict of interest in connection with this article. For the analysis presented in this document, no special funding has been received.

6 Janushevich OO, DmitrievaLA, Grudianov Al (2012) Periodontitis XXIcentury. Moscow: Medical informational agency.

7 Michalowicz BS, Diehl SR, Gunsolley JC (2000) Evidence of a substantial genetic basis for risk of adult periodonti- tis. J Periodontol 71: 1699-1707.

8 Karimbux NY, Saraiya VM, Elangovan S (2012) Interleukin-1 gene polymor- phisms and chronic periodontitis in adult whites: a systematic review and meta-analyses. J Periodontol 83: 1407-1419.

9 Marja L Laine, Bruno G Loos (2010) Gene polimorphisms in Chronic Periodontitis. Int J Dent.

10 Gileva OS, Bondarenko EA, Gibadullina NB (2008) Topical phagotherapy in the complex treatment of gingivitis and periodontitis. J Medical Perm 28: 87-93.

11 Pashkova GS, Volkov EA, Nikitin VV (2013) The use of a bacteriophage- 
based agent in the complex treatment of infectious-inflammatory periodontal diseases. J Russian Dental J 2013: 21-25.

12 Michalowicz BS (1994) Inherited phagocytic cell deficiencies appear to confer risk for prepubertal periodontitis. J Periodontol 65: 479-488.
13 Grudianov Al, Aleksandrovskaja IJ (2010) Planning of treatment for periodontal disease. Moscow: Medical Informational Agency.

14 Janushevich OO, DmitrievaLA, Grudianov Al (2012) Periodontitis XXIcentury. Moscow: Medical informational agency.

15 Volf GF, Rateitschak EM (2008) Parodontology. Moscow: Medpressinform. 